\title{
Usage of C-Reactive Protein Testing in the Diagnosis and Monitoring of Psoriatic Arthritis (PsA): Results from a Real-World Survey in the USA and Europe
}

\author{
A. Ogdie $\cdot$ W. Tillett $\cdot$ N. Booth (D) $\cdot$ O. Howell $\cdot$ A. Schubert $\cdot$ \\ S. Peterson · S. D. Chakravarty · L. C. Coates
}

Received: September 22, 2021 / Accepted: December 17, 2021 / Published online: January 15, 2022

(C) The Author(s) 2022

\section{ABSTRACT}

Introduction: C-reactive protein (CRP) is an important non-specific marker of both acute and chronic inflammation and can be elevated in patients with psoriatic arthritis (PsA). However, the use of CRP testing in the management of PsA can vary. This study investigated how CRP testing is implemented in real-world clinical practice for disease management of PsA.

Methods: A point-in-time survey of rheumatologists and dermatologists and their next six

\section{A. Ogdie}

Perelman School of Medicine, Philadelphia, USA

W. Tillett

University of Bath, Bath, UK

N. Booth $(\varangle) \cdot$ O. Howell

Adelphi Real World, Bollington, UK

e-mail: nicola.booth@adelphigroup.com

A. Schubert

Janssen-Cilag, Warsaw, Poland

S. Peterson

Janssen Global Services, LLC, Raritan, USA

S. D. Chakravarty

Janssen Scientific Affairs, LLC, Titusville, USA

S. D. Chakravarty

Drexel University College of Medicine,

Philadelphia, USA

L. C. Coates

University of Oxford, Oxford, UK consulting patients with PsA was conducted in France, Germany, Italy, Spain, UK (EU5), and the USA between June and August 2018. Use of CRP testing was obtained by asking the physician to state (yes/no) whether CRP was used to aid PsA diagnosis and/or to monitor the patient's disease activity. The number of CRP tests conducted in the last 12 months for each patient enrolled was provided.

Results: Data were collected for 2270 patients (USA, $n=595$; EU5, $n=1675$ ). In the EU5, CRP testing was conducted to aid diagnosis in $78.7 \%$ of patients (vs. $43.4 \%$ in USA) and CRP was used to monitor disease activity in $72.0 \%$ (vs. $34.6 \%$ in USA). The majority (80.9\%) of patients in the EU5 had at least one CRP test in the last 12 months compared to $42.9 \%$ in the USA. Patients treated by rheumatologists (vs. dermatologists) were at least $50 \%$ more likely to have CRP tested for monitoring purposes, this difference being most pronounced in the USA. In the EU5, CRP testing was conducted a mean \pm standard deviation of $2.7 \pm 1.7$ times during the last 12 months, versus $2.0 \pm 1.4$ in the USA.

Conclusions: CRP was more commonly used for the diagnosis and monitoring of PsA in Europe compared to the USA and was more commonly ordered by rheumatologists than dermatologists. In the absence of a better serum biomarker of inflammation, more data are needed to understand how CRP testing should be used in the diagnosis and management PsA. 
Keywords: Psoriatic arthritis; Inflammation; Creactive protein; Testing patterns; Real world evidence

\section{Key Summary Points}

Why carry out this study?

While C-reactive protein (CRP) levels can be elevated in patients with psoriatic arthritis (PsA), the use of CRP testing in the management of PsA can vary

This study investigated how CRP testing for the diagnosis and monitoring of PsA is implemented in real-world clinical practice

\section{What was learned from the study?}

CRP testing was more commonly used for diagnosis and monitoring of PsA in Europe than in the USA

CRP testing was more commonly used by rheumatologists than dermatologists

In the absence of a better serum biomarker of inflammation, more data are needed to understand how CRP testing should be used in the diagnosis and management PsA

\section{INTRODUCTION}

C-reactive protein (CRP) is an important nonspecific marker of both acute and chronic inflammation. However, while CRP values are known to be elevated in a proportion of patients with psoriatic arthritis (PsA) [1], and are associated with worse disease severity and symptomatic burden, many patients with PsA do not have raised CRP levels, despite having active inflammation.

CRP testing is inexpensive and accessible, however, its utility as a marker of interest in inflammatory conditions including PsA can vary [2]. There is relative uncertainty surrounding its reliability, validity as a marker of disease activity, and its prognostic value. Despite this uncertainty, CRP testing is a part of several key composite outcome measures, including the American College of Rheumatology Response Criteria, the primary outcome in PsA clinical trials, and the Disease Activity of Psoriatic Arthritis (DAPSA) score, as well as rheumatoid arthritis outcome measures commonly employed in PsA (i.e., Disease Activity Score-28 [DAS28]) [3]. There is wide variability in the use of CRP across physicians monitoring psoriatic disease, including in PsA management, and variable ability to have laboratory values at the time of the clinic visit. The objective of this study was to investigate how CRP testing is implemented in real-world clinical practice for disease management of PsA.

\section{METHODS}

Data were drawn from the Adelphi PsA Disease Specific Programme (DSP ${ }^{\mathrm{TM}}$ ), a large, multinational point-in-time survey of rheumatologists and dermatologists and their consulting patients in a real-world clinical setting. This survey was conducted in Europe (France, Germany, Italy, Spain, UK; i.e., EU5) and the USA between June and August 2018. A complete description of the survey methodology has previously been published and validated [4]. A geographically diverse sample of physicians were recruited across different sites and settings in order to generate a sample reflective of current clinical practice across all regions and territories.

Physicians were eligible to participate if they had 3-30 years of experience and were personally and directly responsible for the management of patients with PsA. Physicians completed patient record forms for up to their next six consecutive consulting patients; these visits could be for diagnosis or monitoring purposes, but were all related to the management of PsA. Patients were eligible for inclusion if they visited a participating physician, had a diagnosis of PsA, were aged $\geq 18$ years, and were not currently involved in a clinical trial. There was no restriction by treatment received. 
Completion of these forms was undertaken through consultation of existing patient clinical records, as well as the judgement and diagnostic skills of the respondent physician, consistent with decisions made in routine clinical practice. Missing data were not imputed; therefore, the base of patients for analysis could vary from variable to variable and is reported separately for each analysis.

Use of CRP testing was obtained by asking the physician to state (yes/no) whether CRP was used to aid PsA diagnosis, confirm the patient's PsA, and/or to monitor the patient's PsA, with the selection made according to physician judgement in consultation with the patient's clinical chart history. Where physicians stated use of CRP testing, they were then asked to provide the number of CRP tests conducted in the last 12 months, as well as the reason for conducting the test (either for monitoring or diagnostic purposes). Currently prescribed disease-modifying antirheumatic drug (DMARD) treatment usage was also recorded. Severity of disease was reported by physicians who classified their patients as having mild/moderate/severe disease based on their own judgement.

Data collection was undertaken in line with European Pharmaceutical Marketing Research Association guidelines [5], and as such did not require ethics committee approval. However, ethical approvals were sought and granted through the Freiburg Ethics Commission in Europe (study code: 02018/1077) and Western Institutional Review Board in the USA (study number: 1183030). Each survey was performed in full accordance with relevant legislation at the time of data collection, including the US Health Insurance Portability and Accountability Act 1996 [6], and Health Information Technology for Economic and Clinical Health Act legislation [7]. Data were collected in such a way that patients and physicians could not be identified directly; all data were aggregated and de-identified before receipt, and informed consent was given.

The analysis involved descriptive statistics and, where appropriate, the proportion of patients (number and percentage) or mean values with standard deviation (SD) were reported.

\section{RESULTS}

Data were collected for 2270 patients with PsA (USA, $n=595$; EU5, $n=1675$ ), with an even split between those treated by dermatologists $(n=1140)$ and rheumatologists $(n=1130)$. The mean age \pm SD was $48.6 \pm 13.3,90 \%$ of patients were Caucasian, and $46 \%$ were female. Three quarters $(75 \%)$ of patients were classified with mild disease, with $25 \%$ of patients having moderate/ severe disease (Table 1 ). The mean body mass index $( \pm \mathrm{SD}$ ) was $26.8 \pm 4.7$ (EU5, $26.3 \pm 4.3 \mathrm{~kg} / \mathrm{m}^{2}$; USA, $28.1 \pm 5.5 \mathrm{~kg} / \mathrm{m}^{2}$ ), $20.3 \%$ of patients were smokers (EU5, 24.3\%; USA, $9.5 \%$,), and $58.2 \%$ were working full-time (EU5, 55.6\%; USA, 65.3\%).

Regarding treatment use, just over half $(54.2 \%)$ of all patients in the USA and EU5 received a biologic DMARD (bDMARD), 11.1\% of patients received a targeted synthetic DMARD (tsDMARD; EU5, 7.2\%; USA, 21.8\%), and $36.8 \%$ received a conventional synthetic DMARD (csDMARD; EU5, 41.7\%; USA, 23.0\%) at the time of data collection (Table 1). Of those receiving csDMARDs, $14.9 \%$ were in combination with b/tsDMARDs (EU5, 16.3\%; USA, $10.9 \%$ ) and $21.9 \%$ were as the highest level of treatment (EU5, 25.4\%; USA, 12.1\%). On average, patients visited a physician 6.5 times a year (mean \pm SD visits in past 12 months: EU5, $7.0 \pm 6.3$; USA, $5.0 \pm 3.6$ ).

Use of CRP testing varied between regions, with the majority $(80.9 \%)$ of patients with PsA in the EU5 having at least one CRP test in the last 12 months, compared with $42.9 \%$ in the USA. On average over the past 12 months, CRP testing was conducted more frequently in the EU5 (mean \pm SD: $2.7 \pm 1.7$ times) compared to the USA ( $2.0 \pm 1.4$ times) (Table 1$)$. Use of CRP testing also varied by physician specialty. A greater number of rheumatologists than dermatologists used CRP testing to aid in diagnosis ( 82 vs. $57 \%$ ), to confirm PsA diagnosis (44 vs. $27 \%)$, and to monitor PsA (78\% vs. $46 \%)$; however, the proportions of reported reasons for testing between physician specialities were similar (Fig. 1). The differences previously noted in CRP testing usage between regions was also evident across physician specialities, with 
Table 1 Patient demographic and clinical characteristics

\begin{tabular}{|c|c|c|c|}
\hline Patient demographic and clinical characteristics & Overall $(n=2270)$ & EU5 $(n=1675)$ & USA $(n=595)$ \\
\hline Patient seen by rheumatologist, $n$ (\%) & $1130(49.8)$ & $834(49.8)$ & $296(49.7)$ \\
\hline Age, years, mean $[S D]$ & $48.6[13.3]$ & $48.1[13.1]$ & $50.0[13.5]$ \\
\hline Female, $n(\%)$ & $1047(46.1)$ & $774(46.2)$ & $273(45.9)$ \\
\hline BMI, $\mathrm{kg} / \mathrm{m}^{2}$, mean $[\mathrm{SD}]$ & $26.8[4.7]$ & $26.3[4.3]$ & $28.1[5.5]$ \\
\hline Caucasian, $n(\%)$ & $2051(90.4)$ & $1551(92.6)$ & $500(84.0)$ \\
\hline Current smoker, $n(\%)$ & $403(20.3)$ & $352(24.3)$ & $51(9.5)$ \\
\hline \multicolumn{4}{|l|}{ Employment status, $n$ (\%) } \\
\hline Working full-time & $1271(58.2)$ & $894(55.6)$ & $377(65.3)$ \\
\hline \multicolumn{4}{|l|}{ Patient insurance type, $n(\%)$} \\
\hline GesetzlicheKrankenversicherung (Germany) & & $335(20.0)$ & \\
\hline Sistema Nacional de Salud (Spain) & & $333(19.9)$ & \\
\hline Servizio Sanitario Nazionale (Italy) & & $305(18.2)$ & \\
\hline National Health System (UK) & & $303(18.1)$ & \\
\hline PUMa + CMU-C, mutuelle or assurance privée (France) & & $116(6.9)$ & \\
\hline Protection Universelle Maladie (PUMa) (France) & & $87(5.2)$ & \\
\hline Commercial insurance & & & $405(68.1)$ \\
\hline Medicare & & & $96(16.1)$ \\
\hline Health insurance exchange plan & & & $40(6.7)$ \\
\hline Medicaid (or equivalent) & & & $26(4.4)$ \\
\hline Other & & $196(11.7)$ & $28(4.7)$ \\
\hline \multicolumn{4}{|l|}{ Current disease severity, $n$ (\%) } \\
\hline Mild & $1702(75.0)$ & $1253(74.8)$ & $449(75.5)$ \\
\hline Moderate/severe & $568(25.0)$ & $422(25.2)$ & $146(24.5)$ \\
\hline \multicolumn{4}{|l|}{ Current treatment, $n(\%)$} \\
\hline Receiving bDMARD & $1231(54.2)$ & $910(54.3)$ & $321(53.9)$ \\
\hline Receiving tsDMARD & $251(11.1)$ & $121(7.2)$ & $130(21.8)$ \\
\hline Receiving csDMARD & $835(36.8)$ & $698(41.7)$ & $137(23.0)$ \\
\hline In combination with $\mathrm{b} / \mathrm{ts} \mathrm{DMARD}$ & $338(14.9)$ & $273(16.3)$ & $65(10.9)$ \\
\hline As highest level of treatment & $497(21.9)$ & $425(25.4)$ & $72(12.1)$ \\
\hline Receiving opioid & $55(2.4)$ & $29(1.7)$ & $26(4.4)$ \\
\hline
\end{tabular}


Table 1 continued

\begin{tabular}{llll}
\hline Patient demographic and clinical characteristics & Overall $(\boldsymbol{n}=\mathbf{2 2 7 0})$ & EU5 $(\boldsymbol{n}=\mathbf{1 6 7 5})$ & USA $(\boldsymbol{n}=\mathbf{5 9 5})$ \\
\hline $\begin{array}{l}\text { Total number of HCP visits in last } 12 \text { months, mean }[\mathrm{SD}] \\
\text { Frequency of CRP testing }\end{array}$ & $6.5[5.8]$ & $7.0[6.3]$ & $5.0[3.6]$ \\
Patients with $\geq 1$ CRP in last 12 months, $n(\%)$ & $1610(70.9)$ & $1355(80.9)$ & $255(42.9)$ \\
Number conducted in last 12 months, mean [SD] & $2.5[1.7]$ & $2.7[1.7]$ & $2.0[1.4]$ \\
\hline
\end{tabular}

bDMARD Biologic DMARD, csDMARD conventional synthetic DMARD,CRP C-reactive protein, DMARD diseasemodifying antirheumatic drug, $H C P$ healthcare provider, $S D$ standard deviation, tsDMARD targeted synthetic DMARD

uptake of testing by rheumatologists and dermatologists to aid diagnosis, confirm PsA diagnosis, and monitor PsA all being lower in the USA than in the EU5 (Fig. 1).

\section{DISCUSSION}

C-reactive protein is an accessible marker of non-specific inflammation, and CRP testing and can be used for monitoring PsA. However, while elevated CRP levels are associated with worse symptomatic burden in some patients and accelerated disease progression [1], CRP is not always elevated in patients with PsA and active disease. We found that the use of CRP in clinical practice varies depending on physician specialty and region, likely reflecting lack of standardization in the use of CRP testing for PsA management.

The relationship between systemic inflammation and CRP is complex, with CRP levels associated with the risk for cardiovascular disease, diabetes, metabolic syndrome, pulmonary diseases, and depression [8]. Adiposity has also

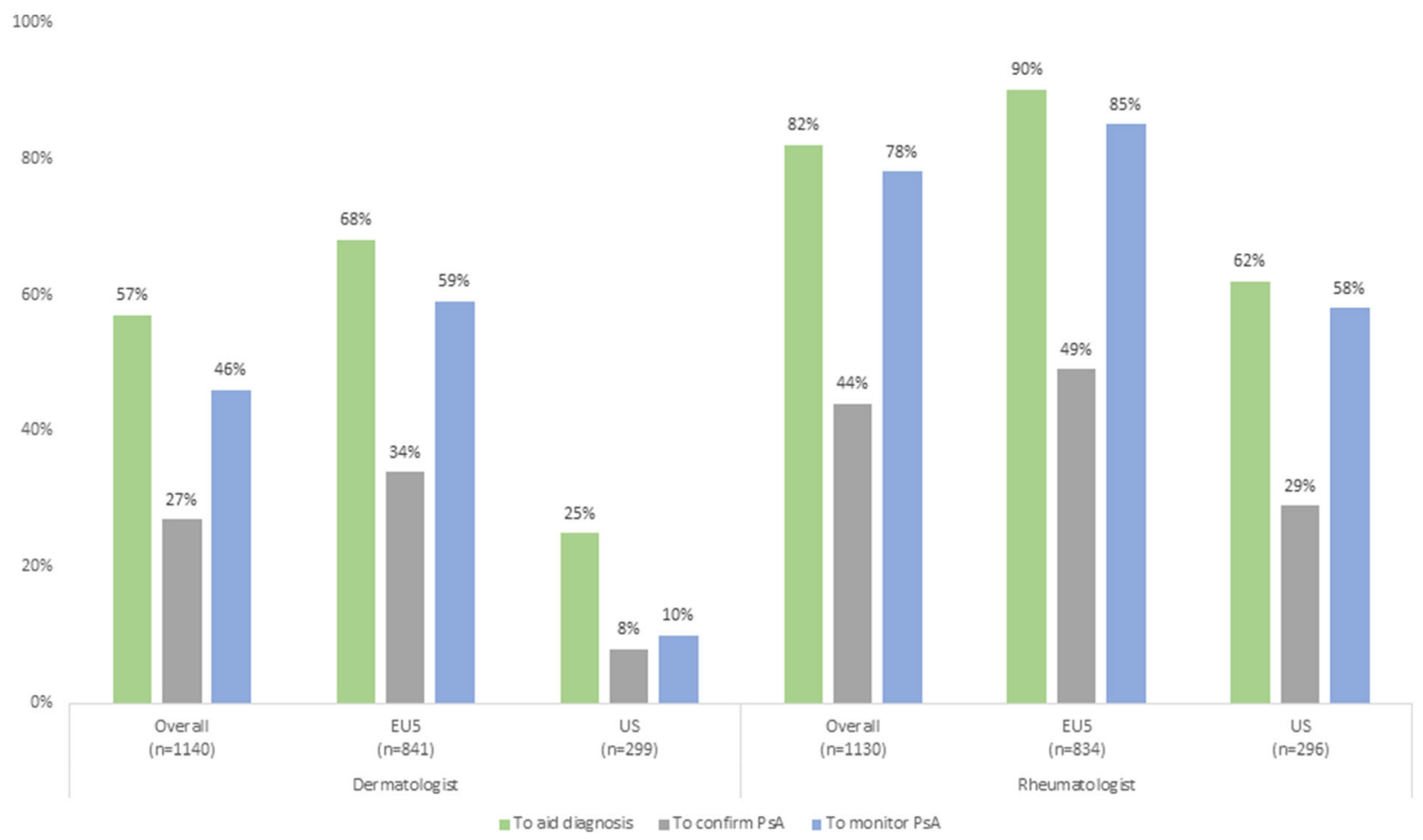

Fig. 1 Purpose of CRP tests conducted, by physician speciality 
been shown to be associated with elevated CRP levels [9]; the association with obesity and both psoriasis and PsA is well established [10], and obesity may be related to the transition from psoriasis (PsO) to PsA [11]. All of these may explain why interpreting CRP levels in patients with inflammatory conditions, in particular around diagnosis, monitoring, disease progression, and risk stratification, can be challenging.

In psoriatic disease, CRP levels have been associated with psoriasis severity (i.e., patients with Psoriasis Area and Severity Index score $>$ 12 on average had a higher CRP) [12]. CRP may also help differentiate PsA from psoriasis without PsA [13]. Among patients with established PsA, studies have found that elevated CRP levels at baseline are associated with a higher likelihood of developing radiographic damage over time, indicative of accelerated disease progression $[14,15]$. Additionally, patients with consistently normal CRP levels over time have generally been found to have milder disease or less structural damage over time [16]. Finally, using data from randomized controlled trials, CRP levels may also be useful for assessing therapeutic response in PsA, though these data are limited [3, 12, 17].

CRP testing is frequently used in the management of rheumatoid arthritis, a related disorder that is often assessed and treated similarly. It is thus not surprising that the use of CRP testing in clinical practice was more common amongst rheumatologists compared to dermatologists in this study, which may be in part due to reasons of awareness, education, and comfort with the CRP testing process or time available for checking and reviewing the results of laboratory tests. It could be argued that physicians undertake differing roles in the diagnosis and management of PsA, with rheumatologists more orientated towards diagnosis and dermatologists towards management; however, we would still expect both specialities to assess CRP levels as part of a broader set of tests. While we do not report on the reason for patient visit to the physician at the point of recruitment, we do report on the number of tests undertaken over the preceding 12-month period, as well as the reasons these tests were performed (either for diagnostic or monitoring purposes), with the relative proportions of reported reasons for testing between physician specialities being broadly similar.

Additionally, use of CRP testing was more common in Europe than in the USA. This may be related to challenges in getting laboratory values in the USA given different electronic medical records and differing insurance coverage of laboratory venues. Patients in the USA had a range of insurance statuses, which could impact on the reduced level of testing observed in this region. While CRP testing is relatively inexpensive, the therapeutic options available may not be reimbursed or affordable if the patient has no insurance coverage, and thus physicians and their consulting patients in the USA may conclude that CRP testing is not worthwhile or that it is not required to be conducted as frequently as we observed in Europe, given the potential for considerable out-ofpocket expense.

\section{LIMITATIONS}

Physicians were asked to provide data on their next six consulting patients, in order to mitigate against selection bias. Physicians completed the record forms with access to the patient's historical medical records, with data collected at the time of consultation to limit recall bias. However, while the sample is representative of the consulting patient population, patients visiting their physician may be more severely affected than those who do not consult their physician as frequently. Data collection relied on the judgement and diagnostic skills of the respondent physician, which meant that responses to questions could be open to interpretation; however, this is consistent with decisions made in real world clinical practice.

\section{CONCLUSION}

The use of CRP testing for diagnosis and monitoring of PsA in clinical practice varied widely, with more frequent use across Europe than in the USA, and greater uptake by rheumatologists than dermatologists. Despite its limitations, 
CRP remains the only currently available biomarker of inflammation, and should be used alongside other measures of disease activity, such as joint counts, to either confirm diagnosis or monitor changes in disease status. Additional studies are needed to identify other potential biomarkers in PsA and to better understand the interpretation and use of CRP testing in the diagnosis and management of PsA, not only in the USA and Europe but also in emerging markets where the use of CRP testing may not be as established.

\section{ACKNOWLEDGEMENTS}

Funding. Data collection was undertaken by Adelphi Real World as part of an independent survey. All data that support the findings of this study are the intellectual property of Adelphi Real World. Janssen Research and Development, LLC, were one of multiple subscribers to the survey, and did not influence the original survey through either contribution to the design of questionnaires or data collection. Janssen Research and Development, LLC, funded the journal's Rapid Service Fee. Laura C Coates is funded by a National Institute for Health Research Clinician Scientist award and supported by the National Institute for Health Research (NIHR) Oxford Biomedical Research Centre (BRC). The views expressed are those of the author(s) and not necessarily those of the NHS, the NIHR or the Department of Health.

Medical Writing, Editorial and Other Assistance. Medical writing support under the guidance of the authors was provided by Gary Sidgwick PhD of Adelphi Real World, in accordance with Good Publication Practice (GPP3) guidelines [18].

Authorship. All authors were involved in: (1) conception or design, or analysis and interpretation of data; (2) drafting and revising the article; (3) providing intellectual content of critical importance to the work described; and (4) final approval of the version to be published. Therefore, they meet the criteria for authorship in accordance with the International Committee of Medical Journal Editors (ICMJE) guidelines [19]. In addition, all named authors take responsibility for the integrity of the work as a whole and have given their approval for this version to be published.

Author Contributions. O Howell, N Booth, A Schubert, and S Peterson contributed to the study conception and design. $\mathrm{N}$ Booth and $\mathrm{O}$ Howell performed the data analysis, and all authors contributed to the interpretation of the data. All authors provided intellectual content of importance to the work described, critically reviewed this and prior drafts of the manuscript and gave their final approval of this version to be published.

Prior Presentation. The data in this study were previously presented as a poster at the European League Against Rheumatism (EULAR) online conference in June 2020 (poster number FRI0358).

Disclosures. A Ogdie has received grant/research support from Amgen (to Forward), Novartis (to Penn) and Pfizer (to Penn) Inc, and has been a consultant for AbbVie, Amgen, Bristol-Myers Squibb, Celgene, Corrona, Eli Lilly, Gilead, Janssen, Novartis, Pfizer Inc, and UCB. WR Tillett has received research funding, consulting or speaker fees from: Abbvie, Amgen, Celgene, Eli-Lilly, Janssen, Novartis, Pfizer, and UCB. N Booth and O Howell are employees of Adelphi Real World. S Peterson, A Schubert, and SD Chakravarty are employees of Janssen, LLC. $\mathrm{L}$ Coates has received research funding and/or honoraria from Abbvie, Amgen, BMS, Biogen, Celgene, Galapagos, Gilead, GSK, Janssen, Lilly, Medac, Novartis, Pfizer, and UCB.

Compliance with Ethics Guidelines. Data collection was undertaken in line with European Pharmaceutical Marketing Research Association guidelines [5], and as such did not require ethics committee approval. However, ethical approvals were sought and granted through the Freiburg Ethics Commission in Europe (study code: 02018/1077) and Western Institutional Review Board in the US (study 
number: 1183030). Each survey was performed in full accordance with relevant legislation at the time of data collection, including the US Health Insurance Portability and Accountability Act 1996 [6], and Health Information Technology for Economic and Clinical Health Act legislation [7]. Data were collected in such a way that patients and physicians could not be identified directly; all data were aggregated and de-identified before receipt and informed consent was given.

Data Availability. Data collection was undertaken by Adelphi Real World as part of an independent survey, entitled the Adelphi Psoriatic Arthritis Disease Specific Programme (DSP). The DSP is a wholly owned Adelphi product. All data, i.e., methodology, materials, data and data analysis, that support the findings of this survey are the intellectual property of Adelphi Real World. All requests for access should be addressed directly to Nicola Booth (nicola.booth@adelphigroup.com).

Open Access. This article is licensed under a Creative Commons Attribution-NonCommercial 4.0 International License, which permits any non-commercial use, sharing, adaptation, distribution and reproduction in any medium or format, as long as you give appropriate credit to the original author(s) and the source, provide a link to the Creative Commons licence, and indicate if changes were made. The images or other third party material in this article are included in the article's Creative Commons licence, unless indicated otherwise in a credit line to the material. If material is not included in the article's Creative Commons licence and your intended use is not permitted by statutory regulation or exceeds the permitted use, you will need to obtain permission directly from the copyright holder. To view a copy of this licence, visit http://creativecommons.org/licenses/by$\mathrm{nc} / 4.0 /$.

\section{REFERENCES}

1. Beygi S, Lajevardi V, Abedini R. C-reactive protein in psoriasis: a review of the literature. J Eur Acad Dermatol Venereol. 2014;28(6):700-11.

2. Elmamoun M, Leung YY, O'Sullivan D, Steinkoenig I, Chandran V, Gladman DD, et al. Using acutephase reactants to inform the development of instruments for the updated psoriatic arthritis core outcome measurement set. J Rheumatol. 2019;46(3):266-73.

3. Ogdie A, Coates LC, Mease P. Measuring outcomes in psoriatic arthritis. Arthritis Care Res (Hoboken). 2020;72(Suppl 10):82-109.

4. Anderson P, Benford M, Harris N, Karavali M, Piercy J. Real-world physician and patient behaviour across countries: disease-specific programmes-a means to understand. Curr Med Res Opin. 2008;24(11):3063-72.

5. European Pharmaceutical Market Research Association (EphMRA). Code of Conduct. 2019. https:// www.ephmra.org/standards/code-of-conduct/. Accessed Sep 2019.

6. US Department of Health and Human Services. Summary of the HIPAA Privacy Rule 2003. 2003. http://www.hhs.gov/sites/default/files/ privacysummary.pdf. Accessed Sep 2019.

7. Health Information Technology (HITECH). Health Information Technology Act 2009. 2009. https:// www.healthit.gov/sites/default/files/hitech_act_ excerpt_from_arra_with_index.pdf. Accessed Sep 2019.

8. Pope JE, Choy EH. C-reactive protein and implications in rheumatoid arthritis and associated comorbidities. Semin Arthritis Rheum. 2020;51(1): 219-29.

9. Visser M, Bouter LM, McQuillan GM, Wener MH, Harris TB. Elevated C-reactive protein levels in overweight and obese adults. JAMA. 1999;282(22): 2131-5.

10. Armstrong AW, Harskamp CT, Armstrong EJ. The association between psoriasis and obesity: a systematic review and meta-analysis of observational studies. Nutr Diabetes. 2012;2:e54.

11. Green A, Shaddick G, Charlton R, Snowball J, Nightingale A, Smith C, et al. Modifiable risk factors and the development of psoriatic arthritis in people with psoriasis. Br J Dermatol. 2020;182(3):714-20.

12. Asahina A, Umezawa $Y$, Yanaba K, Nakagawa $H$. Serum C-reactive protein levels in Japanese patients 
with psoriasis and psoriatic arthritis: long-term differential effects of biologics. J Dermatol. 2016;43(7):779-84.

13. Cretu D, Gao L, Liang K, Soosaipillai A, Diamandis EP, Chandran V. Differentiating Psoriatic Arthritis From Psoriasis Without Psoriatic Arthritis Using Novel Serum Biomarkers. Arthritis Care Res (Hoboken). 2018;70(3):454-61.

14. van der Heijde D, Gladman DD, FitzGerald $O$, Kavanaugh A, Graham D, Wang C, et al. Radiographic progression according to baseline C-reactive protein levels and other risk factors in psoriatic arthritis treated with tofacitinib or adalimumab. J Rheumatol. 2019;46(9):1089-96.

15. Gladman DD, Mease PJ, Choy EH, Ritchlin CT, Perdok RJ, Sasso EH. Risk factors for radiographic progression in psoriatic arthritis: subanalysis of the randomized controlled trial ADEPT. Arthritis Res Ther. 2010;12(3):R113.

16. Haroon M, Gallaghar P, Ahmad M, FitzGerald O. Elevated CRP even at the first visit to a rheumatologist is associated with long-term poor outcomes in patients with psoriatic arthritis. Clin Rheumatol. 2020;39(10):2951-61.

17. Wu JJ, Rowan CG, Bebchuk JD, Anthony MS. Association between tumor necrosis factor inhibitor (TNFi) therapy and changes in C-reactive protein (CRP), blood pressure, and alanine aminotransferase (ALT) among patients with psoriasis, psoriatic arthritis, or rheumatoid arthritis. J Am Acad Dermatol. 2015;72(5):917-9.

18. Battisti WP, Wager E, Baltzer L, Bridges D, Cairns A, Carswell CI, et al. Good publication practice for communicating company-sponsored medical research: GPP3. Ann Intern Med. 2015;163(6): 461-4.

19. International Committee of Medical Journal Editors (ICMJE). Defining the role of authors and contributors. 2020. http://www.icmje.org/recommend ations/browse/roles-and-responsibilities/definingthe-role-of-authors-and-contributors.html. Accessed Jan 2020. 